\title{
High thermal conductivities of carbon nanotube films and micro-fibres and their dependence on morphology
}

Thurid S. Gspann ${ }^{\mathrm{a}, *}$, Stefan M. Juckes ${ }^{\mathrm{b}}$, John F. Niven ${ }^{\mathrm{b}}$, Michel B. Johnson ${ }^{\mathrm{c}}$, James A. Elliott ${ }^{\mathrm{a}}$, Mary Anne White ${ }^{\mathrm{b}, \mathrm{c}, \mathrm{d}}$, Alan H. Windle ${ }^{\mathrm{a}}$

${ }^{\text {a }}$ Department of Materials Science and Metallurgy, 27 Charles Babbage Road, University of Cambridge, CB3 0FS, United Kingdom.

${ }^{\mathrm{b}}$ Department of Physics and Atmospheric Science, Dalhousie University, Halifax, NS B3H 4R2, Canada.

${ }^{\mathrm{c}}$ Institute for Research in Materials, Dalhousie University, Halifax, NS B3H 4R2, Canada.

${ }^{\mathrm{d}}$ Department of Chemistry, Dalhousie University, Halifax, NS B3H 4R2, Canada.

\begin{abstract}
Thermal conductivity of carbon nanotube (CNT) films and micro-fibres synthesised by floating catalyst chemical vapour deposition was measured by the parallel thermal conductance method. CNT films showed in-plane thermal conductivities of $110 \mathrm{~W} \mathrm{~m}^{-1} \mathrm{~K}^{-1}$. Online condensation into a micro-fibre morphology - a two-dimensional reduction in the transverse plane, including some axial stretching during solvent evaporation - resulted in room-temperature thermal conductivity values as high as $770 \pm 10 \mathrm{~W} \mathrm{~m}^{-1} \mathrm{~K}^{-1}$, which is the highest thermal conductivity reported for CNT bulk materials to date. In specific terms, this matches the maximum thermal conductivity of heattreated carbon fibre, but with a higher onset temperature for Umklapp scattering processes (300 K rather than $150 \mathrm{~K}$ ). We selected four sample types to investigate effects of alignment, purity, and single- or multi-wall character on their thermal conductivity. For both the electrical and thermal conductivity of as-spun material, i.e. without any post-synthesis treatment, we show that the density and quality of CNT bundle alignment are still the predominant factors affecting these properties, outweighing the influence of single- or multi-walled character of the nanotubes. This raises the promise that, with optimal alignment and junction points, even higher values of thermal conductivity are achievable for macroscopic CNT fibres.
\end{abstract}

\footnotetext{
*Corresponding author. Tel: +44 (0)1223 334335. E-mail: tsg28@cam.ac.uk (Thurid Gspann)
} 


\section{Introduction}

Modern technology continues to test the limits of materials science, demanding increasingly lightweight, high performance, energy-efficient and cost-effective materials. For decades, nanoscale materials have been touted as one way to meet these demands, and carbon nanotubes (CNTs) are amongst the most promising and well-studied of these at the level of individual tubes. However, in practice, bulk CNT materials are required, and their physical and microstructural properties must be understood and optimized as, overall, they are the factors inhibiting the utilization of the superlative properties of individual CNTs.

We consider floating catalyst CVD synthesis, known as direct spinning, to be intrinsically suitable for cost-effective scale-up of CNT fibre production for the following reasons: (1) amongst the main CNT production processes, namely arc discharge, laser ablation, and chemical vapour deposition, CVD processes are most promising for CNT mass production [1], (2) the single-step process is most similar to the production of carbon black [2], rather than multi-step processes employed for polymer fibre [3] or carbon fibre [4] [5] production, and thus inherently inexpensive; (3) the direct spinning process is continuous and substrate-free, not a batch process. However, the disadvantages of this process lie in the large number of interdependent parameters that must be optimised in order to maximise the yield and quality of the product in terms of its mechanical, electrical and thermal properties.

Compared to their individual constituents [6] [7] [8], CNT bulk materials such as films (2D), forests (3D) or fibres (1D), suffer losses of several orders of magnitude in electrical conductivity because of (1) the presence of impurities and defects, which cause electron scattering and contact resistance, and (2) the insufficient alignment and CNT overlap length leading to increased junction resistance. An extensive review of conduction dependence on defects, nanotube packing and alignment and the state of electrical conductivity of bulk materials such as CNT fibres can be found in [9]. As for electrical and mechanical properties, the thermal conductivities of carbon nanotube bulk materials to date are considerably less than experimental values obtained for individual nanotubes and have provoked considerable interest particularly over the last five years.

In general, carbon-based materials are very attractive due to the combination of their exceptional thermal properties accompanied by their low densities. The best representatives of the family are diamond (with thermal conductivity up to $3320 \mathrm{~W} \mathrm{~m}^{-1} \mathrm{~K}^{-1}$ for nearly isotopically pure diamond [10]) and heat-treated carbon fibre (with thermal conductivity up to $2600 \mathrm{~W} \mathrm{~m}^{-1} \mathrm{~K}^{-1}$ [11] [12] [13]). However, both materials are expensive; in the case of carbon fibre this is due to the multi-stage and 
time-consuming production process. The thermal conductivity of isolated individual single-wall CNTs has been reported as among the highest measured values of any known material. With roomtemperature thermal conductivities of $3500 \mathrm{~W} \mathrm{~m}^{-1} \mathrm{~K}^{-1}$ [14] [15], individual SWCNTs experimentally show thermal conductivities greater than that of diamond. Early molecular dynamics simulations predicted values of up to $6600 \mathrm{~W} \mathrm{~m}^{-1} \mathrm{~K}^{-1}$ at room temperature due to the high purity and stiffness of the carbon-carbon bonds leading to long phonon mean free paths [16], with maximum values of $10,000 \mathrm{~W} \mathrm{~m}^{-1} \mathrm{~K}^{-1}$ at $100 \mathrm{~K}$. However, Mingo et al. [17] questioned such ultra-high values and showed that the thermal conductances of SWCNTs might be smaller than previously thought, while the ballistic lengths are orders of magnitude larger. Hence, the values of thermal conductivity measured experimentally are actually in reasonable agreement with theoretical estimates.

In metals, both the thermal and electrical conductivity follow the same mechanisms of electronic conduction, and decrease with increasing temperature due to phonon scattering of the conduction electrons [18]. Pure anisotropic crystalline substances exhibit different thermal conductivities along different crystal axes, due to differences in phonon coupling in different directions. In CNTs, even metallic CNTs, both thermal conductivity and heat capacity of CNTs are dominated by the phononic modes [15] and transport is quasi-one-dimensional. At temperatures below $30 \mathrm{~K}$ [15], the thermal conductivity of CNTs is ballistic. The thermal conductivity, $\kappa$, increases strictly linearly with temperature $(\kappa \sim T)$ and extrapolates to zero at $0 \mathrm{~K}$. The ballistic regime becomes less dominant at higher temperatures as phonon-phonon, phonon-boundary, and phonon-defect scattering become more prevalent [17] [19], and both heat capacity, $C$, and $\kappa$ increase according to a power law $\kappa \sim C \sim T^{\alpha}$, with $\alpha>1$ to 3 depending on the dimensionality of the phonon heat conduction [14] [17] [20] [21] [22]. The thermal conductivity then peaks near 200 to $300 \mathrm{~K}$ [23] [14] [20] [24] due to Umklapp processes, where the excitation of high-frequency phonon modes is offset by the increased phonon scattering probability. Above that peak temperature, phonon-phonon scattering begins to dominate, and $\kappa$ decreases continuously with $\sim T^{-\alpha}$, with $\alpha \sim 1$ to 2 [14]. Noteworthy, this onset for Umklapp processes occurs at significantly higher temperature than for diamond, graphite or carbon fibre (150 K) [23] [16] [13] [11].

Defects in CNTs, including flaws in the tube wall, impurities, bends and kinks can significantly reduce $\kappa$, even at low defect concentrations, as they strongly suppress high-frequency phonon modes [25] [26]. The room-temperature value of the phonon mean free path, $\lambda_{\text {ph }}$, estimated to range 
from $50 \mathrm{~nm}$ to $1.5 \mu \mathrm{m}$ in pristine SWCNTs [27] [20] [15], can be as low as $4 \mathrm{~nm}$ in defective CNTs [14].

In MWCNTs, inter-shell forces mean that heat conduction is no longer purely one-dimensional. On one hand, the larger diameter allows for more phonon modes [28], and the thermal conductance of MWCNTs is higher than the sum of the thermal conductances of SWCNTs corresponding to each wall in MWCNTs, because the bending deformation of a tube protected by an outer tube is significantly less than that of the SWCNT without protection [29] [30]. On the other hand, the thermal conductivity of MWCNTs is smaller than that of a SWCNT corresponding to the outer tube, because the increase of cross-sectional area dominates over the increase of thermal conductance. For bundles, the thermal conductivity decreases with increasing number of CNTs associated with the quenching of phonon modes by neighbouring CNTs and the high thermal resistance at tube-tube junctions. This decrease saturates when the diameter of the bundles reaches around $150 \mathrm{~nm}[30]$.

For CNT bulk materials, the thermal conductivity is reduced, foremost because of their yarn-like structure: aligned bundles laterally connected by interwoven poorly oriented nanotubes lead to relatively low density, while the large surface area causes large losses due to radiation [30].

Tube-tube interactions between CNTs are dominated by van der Waals forces, which essentially vanish for CNTs separated by more than $0.4 \mathrm{~nm}$ [30] [31] [32]. Due to this weak interaction, heat flow between overlapping CNTs is low and the overlap length becomes highly important to determine the thermal resistance between tubes. For electrical conductivity and mechanical properties, CNT length, alignment and thus maximizing the contact between tubes [9] [33] [34] [35] have been shown to be the main limiting factors to approach optimal values. Similarly for thermal conductivity, alignment is of high importance as the heat flow parallel to the CNT axis is far higher than perpendicular to it [23] [36] [37] [38], however, rather than continuous contact, Baughman et al. [30] suggested that the ideal overlap length is $30-35 \mu \mathrm{m}$ to reduce inter-tube scattering and dampen thermal vibrations, while still providing sufficient length to transfer thermal energy between the nanotubes.

CNT yarns produced by either wet-spinning or forest drawing processes typically achieve roomtemperature thermal conductivities of only tens of $\mathrm{W} \mathrm{m}^{-1} \mathrm{~K}^{-1}$ [39], but up to 380 or $635 \mathrm{~W} \mathrm{~m}^{-1} \mathrm{~K}^{-1}$, if treated with acids or iodine-doped, respectively [24].

For heterogeneous materials such as CNT bulk material, which are effectively low-density CNT/air composites (or CNT/vacuum composites during measurements) [40], $\kappa$ can vary 
significantly with the cross-sectional area and density of the CNT bundle network. Experimental measurements of composite materials give a value of apparent thermal conductivity, $\kappa_{\mathrm{app}}$, averaged over the components of the composite. The value of $\kappa_{\text {app }}$ can be viewed as $\kappa$ for a homogeneous material with equivalent physical dimensions. Because of the high porosity of bulk CNT materials, the true cross-section through which heat can flow is significantly smaller than the overall (macroscopic) cross-sectional area. Attempts can be made to correct for the reduced density, such as normalizing the measured values to the ideal density hexagonally packed SWCNTs. However, we report the apparent thermal conductivity without correction for an ideal density, as this is the value that is most relevant to applications.

Similar to specific electrical conductivity, we report thermal conductivity in specific terms normalised by bulk density $(\kappa / \rho)$, but using the linear density [41] to circumvent the necessity for measuring the thickness of films or diameter of fibres, which is the main source of uncertainty in density determination. (A detailed derivation of units can be found in Suppl. Inf.). Furthermore, $\kappa / \rho$ can be directly related to thermal diffusivity, $a\left(a=\kappa\left(\rho C_{\mathrm{s}}\right)^{-1}\right.$ where $C_{\mathrm{s}}$ is specific heat, which is rather independent of the type of CNT).

In this paper, we describe the thermal conductivity of four samples synthesised by the same process, but from different carbon precursors and precursor concentration ratios, which were selected for their difference in bundle alignment, composition of single- or multi-wall CNTs, and amount of carbonaceous bundle coating or cluster impurities comprised of deformed CNTs. We determined the thermal conductivity of these samples, both in absolute and specific terms, and compare the properties of sheet material and micro-fibres, to draw conclusions about the ideal composition of CNT bulk materials for heat conduction.

\section{Experimental methods}

\subsection{CNT Samples Synthesis}

All samples were produced by a direct CNT fibre spinning process using floating catalyst chemical vapour deposition [42] [41] [43]. A gaseous or vapour feedstock of three different hydrocarbon precursors was used, namely methane, toluene and n-butanol, with ferrocene as catalyst, and thiophene as sulphuric growth promoter. All synthesis parameters were optimised for stable and continuous spinning and are summarised in Supp. Inf., Table S1, stating the most important parameters that give control over the multi-parameter process, for CNT type and 
orientation. The inter-parameter dependences of the floating catalyst CVD were discussed in [44]. If the reactant concentration in the reaction zone (given as precursor input and hydrogen flow) is high, the spinnability is in general high, but the occurrence of undesired CNT species or impurities is high as well. If the iron precursor concentration is high, then so is the collision of catalyst particles, which leads to particle agglomeration and hence growth of multi-wall CNTs. High concentration of carbon precursor leads to more contamination with amorphous carbon, and high content sulfuric species leads to growth of multi-wall rather than single-wall CNTs and finally to catalyst poisoning [42] [45] [46]. On the other hand, if the reactant concentration is very low, the sparse distribution of CNTs cannot provide sufficient mechanical entanglement for spinning. The effects of hydrogen throughput and reactor tube diameters have been discussed in [47] and [42]. Operating at high synthesis temperature results in a very fast nanotube growth rate and the long nanotubes formed entangle in form of a gas based gel (aerogel). Similar to polymer strand alignment in polymer fibre production, though in gas phase rather than liquid, the alignment depends on the relationship between the total gas flow in the reactor and the draw speed of the winder, and both are given in Table S1.

All samples were tested as-spun, without any post-synthesis treatments for purification, functionalisation or doping. CNT films were produced by continuously spinning onto a single winder, laying down a dense film of aligned CNT bundles. After spinning, the samples were sprayed with acetone ( $>99.5 \%$ purity) to condense the CNT networks into dense films (condensation process due to surface tension effects described in [48] [49]), and laser-cut into the geometries required for thermal and electrical conductivity measurements. CNT micro-fibres were produced by online condensation with acetone vapour above the first winder, and subsequent stretching between the first and a second rotational winder with a winding speed difference of $5 \%$. The uncertainty of relationship between outer fibre diameter and cross-sectional area can be very high since when the fibre is condensed it usually folds into a non-circular shape (Supp. Inf. S5). In the worst case, an estimation of the cross-sectional area by the outside diameter could lead to errors of up to a factor of five. The cross-sections were therefore measured from several focused-ionbeam-cut cross-sections per sample, or altogether bypassed by the use of linear density (see discussion in Supp. Inf. S1). 


\subsection{Measurement Techniques}

\subsubsection{Electrical conductivity}

Electrical resistance measurements were made using a Physical Properties Measurement System (PPMS; Quantum Design, San Diego, CA). AC current (93 Hz) was used to minimize electromagnetic line interference, and small currents, typically $I=0.1 \mathrm{~mA}$, for $0.5 \mathrm{~s}$ under vacuum $\left(<10^{-7} \mathrm{~atm}\right)$ were used to minimize Joule heating. The electrical resistance was measured as a function of temperature from $390 \mathrm{~K}$ to $2.5 \mathrm{~K}$ after being degassed by heating to $390 \mathrm{~K}$. Measurements used a custom-built 4-wire resistance puck for the PPMS which measures two samples simultaneously [50]. Samples were attached to the leads using high-conductivity silver paint (DuPont 4929N) to ensure a good electrical contact. Care was taken to prevent silver paint from wicking along the sample between the leads.

The electrical conductivity was then calculated from $\sigma=R \cdot A / L$, with electrical resistivity $R$, crosssectional area $A$ and length between contacts $L$. The cross-sectional area was measured using a combination of focused ion beam (FIB) milling (Hitachi FB-2000A) and SEM (Hitachi S-4700 FEG) for CNT tapes (details in Supp. Inf. S4).

\subsubsection{Thermal conductivity}

Usually steady-state methods require rigid samples that can support a heater and thermocouples. These requirements make traditional steady-state methods inappropriate for thin and flexible materials, such as CNT tapes and yarns. The parallel thermal conductance (PTC) method is a modified steady-state measurement in which the sample is supported between two brass platforms, ideal for measuring thin and flexible samples. PTC has been used previously to measure the thermal conductivity of CNT yarns [51] and sheets [50] and boron nitride nanotubes [52], and is described in detail elsewhere [51]. PTC has the additional advantage of measuring a relatively large effective length of yarn, typically $80 \mathrm{~mm}$. In comparison, many other thermal conductivity measurement techniques measure much less sample: $1-10 \mathrm{~mm}$ for $3 \omega$ ( [30] [24]); $<10 \mathrm{~mm}$ for T-type probe [39]; < $50 \mathrm{~mm}$ for comparative approaches [53]. Measuring small lengths of sample makes localized defects a possible source of significant uncertainty.

Background $K_{\mathrm{bg}}$ and radiation thermal conductances $K_{\mathrm{rad}}$ were accurately measured and subtracted. The sample's thermal conductance is then given by $K_{\text {sample }}=K_{\text {total }}-K_{\text {bg }}-0.5\left(K_{\text {rad }}-K_{\text {bg }}\right)$. The factor of 0.5 for the radiation contribution has been estimated by considering the differences in 
the thermal gradient across the sample when it is both connected and disconnected from the cold stage [54].

One platform of the PTC sample stage, the 'hot' plate, has a $120 \Omega$ strain gauge heater attached to it, and is connected to the base using cellulose acetate to minimize heat conduction. The second, 'cold' platform, is heat-sinked to the base using a brass strip in order to maximize heat conduction. The temperature difference between the two posts, $\Delta T$, was measured using a Type E differential thermocouple. Laser-cut textile samples were attached to each stand using silver paint (DuPont 4929 N), while, because of their small diameter, micro-fibre samples were attached to the PTC stage by continuously winding the fibres between the two platforms, attached at each contact point by silver paint.

PTC measurements used the PPMS as a cryogenic vacuum chamber. Temperature difference and applied power were measured externally using separate instrumentation controlled by LabVIEW. All measurements were carried out in vacuum $\left(<10^{-7}\right.$ atm) to minimize heat transfer through the atmosphere. Systematic errors in thermal conductivity measurements by PTC are dominated by uncertainty in the CNT sample dimensions, which was on the order of $10 \%$. There is also some uncertainty in the value of $K$ due to the standard deviation of $\Delta T$ and $P$, but this is usually much smaller. However, the background measurement used in this study for the CNT tapes had significant variation in the applied power leading to non-negligible error. This uncertainty was calculated using a linear regression model and is included in all figures as error bars; where applicable it is added to uncertainty from sample dimensions $(90 \%$ confidence interval, using ttest). As mentioned above, presentation of the results as specific thermal conductivity replaces the rather large length/width uncertainty (ca. 10\%) with a much smaller linear density uncertainty.

\subsubsection{Linear Density}

The linear density of online condensed micro-fibres was determined by using the vibroscopic measurement in a Favimat tensile tester (Textechno, Germany). Originally developed for carbon fibres, the technique is based upon measuring the resonant frequency - linked to the sample's mass for the given gauge length - by determining the maximum amplitude of a first order vibration with a laser. For each sample, three strands of $90 \mathrm{~mm}$ gauge length were measured and averaged with a standard deviation less than 5\%. For the CNT films described in this study, the vibroscopic measurement is less suitable due to their rectangular cross section and potentially very different Eigen-frequencies in the $\mathrm{x}$ and $\mathrm{y}$ directions. The mass of film samples of $3 \mathrm{~mm}$ width is sufficiently high to be measured by a Sartorius Ultramicro-Balance with a standard deviation of less than $1 \%$. 


\section{Results and Discussion}

\subsection{Sample Selection}

Out of a large range of as-prepared CNT samples spun by the direct spinning process, characterised by Raman microscopy, scanning and transmission electron microscopy (SEM, TEM), thermo-gravimetric analysis (TGA) and small angle x-ray scattering (SAXS), we selected four samples for thermal conductivity measurements, that show significant differences in bundle alignment, purity and CNT character.

Depending on their carbon precursors and other spinning conditions, hydrogen flow rate and winding speed, the CNT samples differ in CNT type and purity (Raman and TEM, vide infra and Supp. Inf. S2), bundle alignment, presence or absence of cluster impurities (SEM, vide infra), and content of catalyst residue and non-tubular carbonaceous bundle coating (Thermo-gravimetric Analysis, Supp. Inf. S2). Such coating is a common occurrence in CVD processes and usually simply referred to as amorphous carbon. For alcohol spun fibres, it was characterized in [55] as mostly aliphatic oligomers with lower concentrations of alkyl aromatics, although its particular composition would depend on the thermal and catalytic breakdown of the carbon precursor used. Far underestimated if labelled as mere impurity, such carbonaceous coating can in best cases prove to enhance mechanical properties and electrical stability [56]. The SEM and Raman spectra shown below are only an excerpt of the characterisation carried out; further analysis and tensile stressstrain measurements can be found in the Supplementary Information.

According to a high burning temperature in TGA, the high D/G Raman ratio and TEM images, samples spun from methane (Type A) were characterised as predominantly multi-wall CNTs. TGA showed a carbonaceous impurity content of $5.9 \mathrm{wt} \%$ and residual ferrous nanoparticles from unused catalyst $(<2 \mathrm{wt} \%)$, which is lowest compared to the other sample types. However, SEM and TEM showed the material contained some short deformed multi-wall CNTs, and overall only moderate bundle alignment with a FWHM $=0.8$ obtained from 2D FFT SEM image analysis, and a Chebyshev orientation $T_{2}=0.51$ (detailed description of 2D FFT image analysis and SAXS in Supp. Inf. S2). As described previously [42], those samples, even if medium-aligned and free of large impurities or bundle coating, show only mediocre mechanical strength of $0.7 \mathrm{~N}^{-1}$ and stiffness of $40 \mathrm{~N}$ tex $^{-1}$ [42] (Supp. Inf., Figure S6), with tex $=\mathrm{g} \mathrm{km}^{-1}$, and $\mathrm{N}$ tex ${ }^{-1} \equiv \mathrm{GPa}(\mathrm{g} / \mathrm{cc})^{-1}$ [41]. 

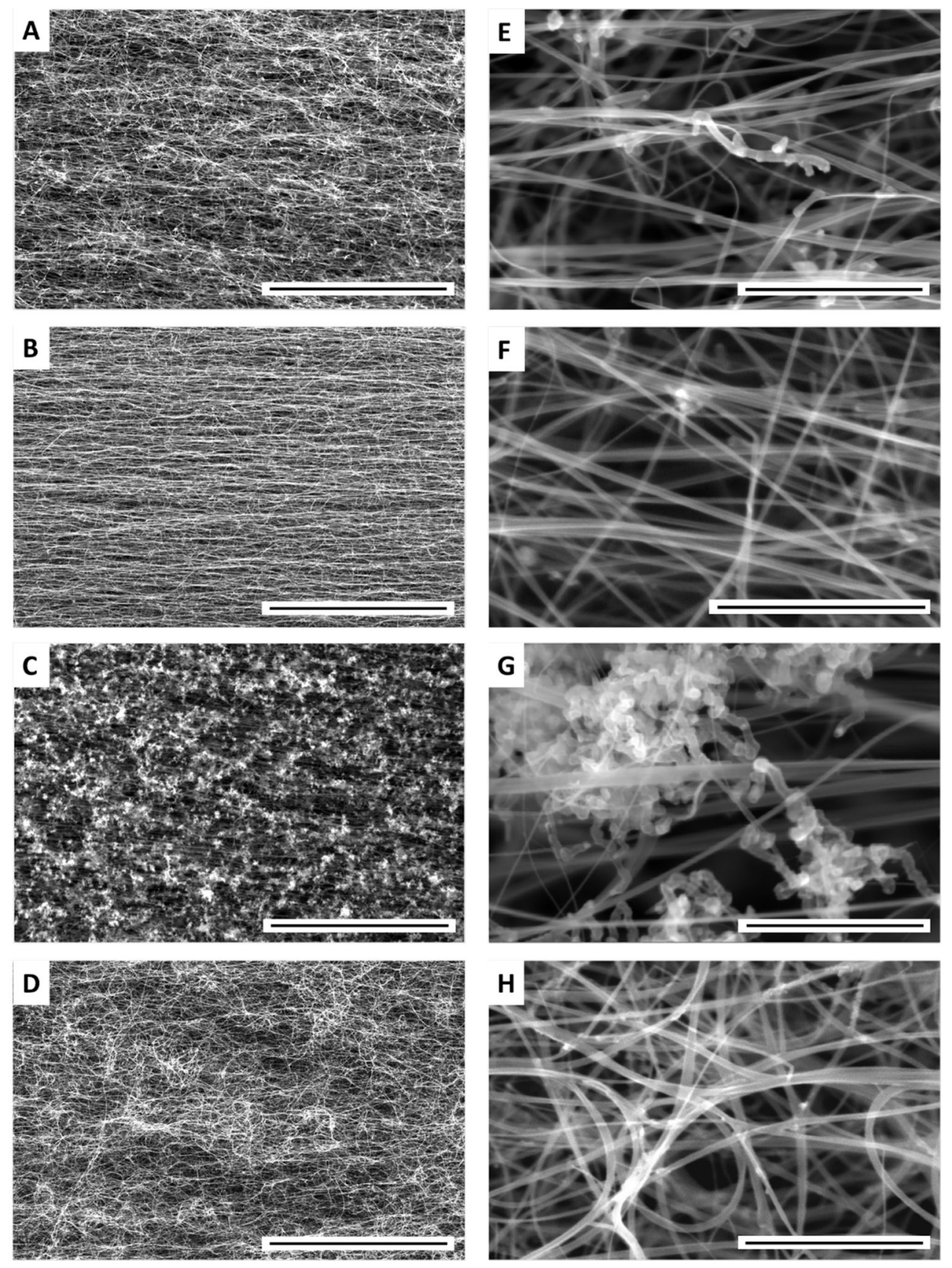

Figure 1: Scanning Electron Micrographs with scale bars of $10 \mu \mathrm{m}$ (A-D) and $500 \mathrm{~nm}(\mathrm{E}-\mathrm{H})$ : CNT films spun from methane - Type A (A,E), toluene - Type B (B,F), n-butanol - Type C $(\mathrm{C}, \mathrm{G})$ showing the typical cluster impurities of curly CNTs, and clean n-butanol spun CNT film - Type D (D,H).

The Type B samples investigated here, spun from toluene, were made using a particular recipe to ensure continuous spinning of relatively thick fibres. The frequent, though not entirely invariant, occurrence of radial breathing modes in Raman when measuring over 25 different positions of the sample indicates a mix of single-walled and multi-walled carbon nanotubes, which was supported by TEM images and TGA showing two main burning events corresponding to two populations of 
CNTs. Typical for Type B, the sample's D peak increases with the amount of bundle coating cosynthesised in the CVD process. A comparatively high amount of such non-tubular carbonaceous coating around the CNT bundles ( $>10 \mathrm{wt} \%$, TGA), together with high alignment with $T_{2}=0.63$ (Figure 1B, SEM image analysis and SAXS in SI) leads to higher internal friction and hence increased tensile strength of $1.7 \mathrm{~N}^{-1} \mathrm{x}^{-1}$ and $80 \mathrm{~N}^{-1} \mathrm{x}^{-1}$ (similar to [42]).

Fibres spun from n-butanol were predominantly composed of single-wall CNTs (Raman, TEM). Given the averaging of 25 measurement positions to give a meaningful characterisation of bulk material, RBMs with significant intensity are shown for samples Type C and D particularly in $785 \mathrm{~nm}$ (Figure 2). At 532 and $633 \mathrm{~nm}$ excitation (Supp. Inf., Figure S3), RBMs are present but their intensity is reduced due to averaging. TGA and TEM show that both sample types were very pure in the sense of absence of bundle coating.

However, in typical n-butanol spun samples (Type C), we observed cluster impurities constructed of highly deformed, curly CNTs (Figure 1C, and TEM in Supp. Inf. Figure S2) grown from oversized catalyst particles and the residual iron oxide content of Type $\mathrm{C}$ samples was typically in the order of $20 \mathrm{wt} \%$. Despite the large amount of cluster impurities, its Raman spectrum (Figure 2) suggests high quality of CNTs and mainly single-walled character. Due to resonance effects the single-wall CNTs dominate the Raman spectra, and other contributions such as external impurities or other non-resonant CNTs with lower intensities would be masked in the signal. Although the load-bearing bundles are straightened during spinning, the cluster impurities impede the bundles from having ideal contact along their length. In this case, 2D FFT analysis of SEM images does not reflect the alignment of the load bearing CNT bundles, as it gives an average of orientations, no matter whether the elements are connected or not, which is dominated by the presence of said cluster impurities. SAXS gives a bundle orientation of $T_{2}=0.49$, which is almost as high as for Type A.

Along the same line of thought as presented in [42], it is possible to produce samples of extraordinary purity (Type D) by using a very steep carrier gas flow profile. The purity and high crystallinity of the sample was characterised by a particularly low D/G Raman peak ratio for asspun samples, and supported by SEM, TEM and TGA analysis (Figures 2, S1 and S2). However, these particularly pure samples are mechanically far inferior to Type B, which enforces a slower winding speed relative to the hydrogen flow through the reactor to ensure continuous spinning, which in turn leads to a lower degree of bundle alignment $\left(T_{2}=0.43\right)$. 


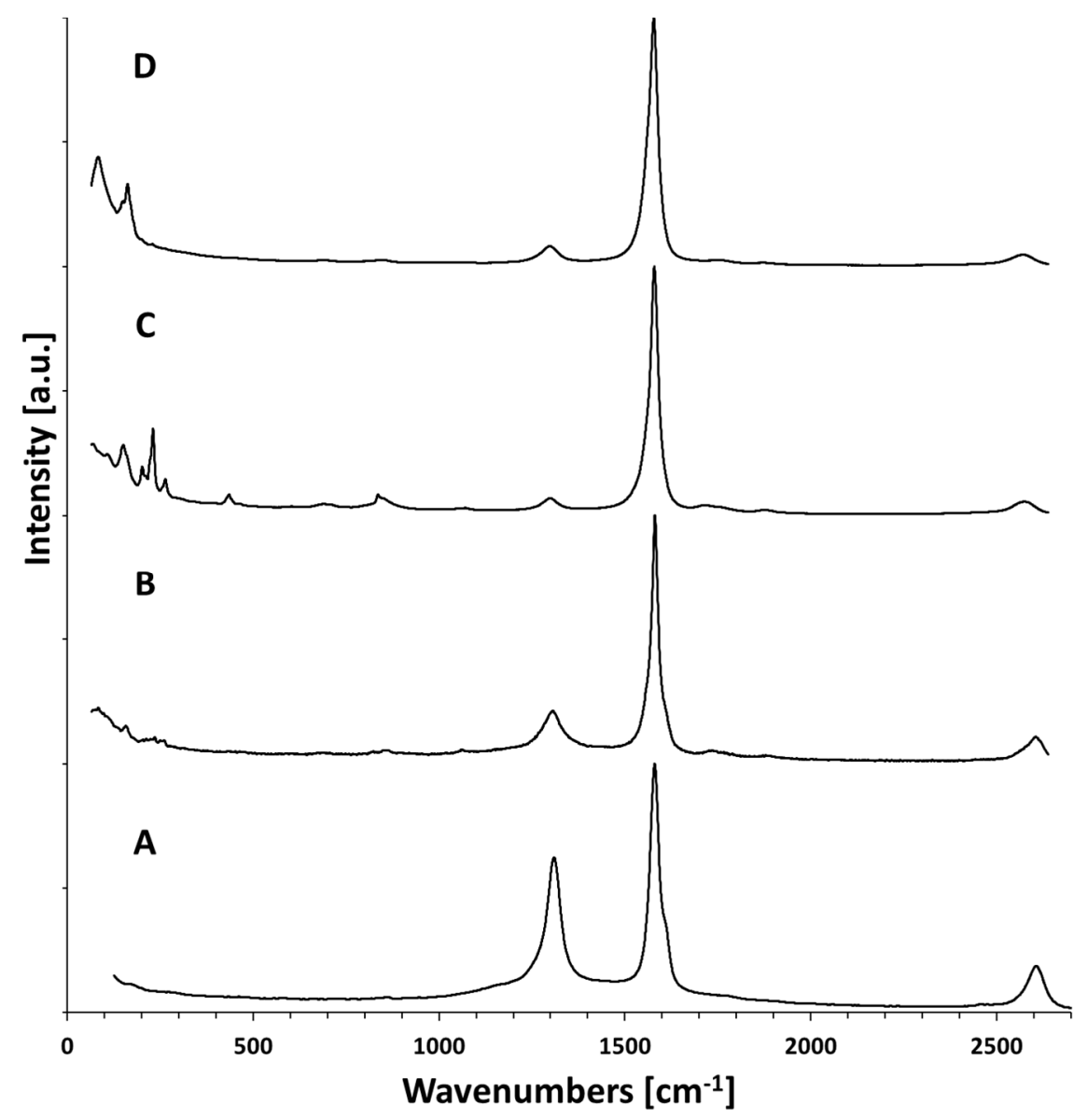

Figure 2: Raman spectra with excitation wavelength $785 \mathrm{~nm}$, normalised to the $\mathrm{G}$ peak. The spectra are averaged over 25 data points each and presented without baseline correction. A, B, C and D refer to the sample type, as described in the caption to Figure 1.

Apart from the mostly multi-walled sample spun from methane, all Raman spectra in Figure 2 show a very low D peak, considering that the materials are presented 'as-spun' without any posttreatments for purification. SEM images show that the most obvious difference between the samples is in their degree of alignment, which drastically decreases for samples spun from n-butanol (Figure 1D). Figure S7 shows the samples in a 3D matrix covering carbon nanotube bundle alignment, predominance of single-wall or multi- wall tubes, and a characteristic value for purity reflecting both structural impurities and bundle coating, which depicts how the samples were selected to cover a range of morphological properties.

\subsection{Electrical Conductivity of CNT Films}

The electrical resistivity was measured as a function of temperature for film samples of all four sample types. It is generally accepted that the main resistance in CNT bulk materials is due to 
junctions between the CNTs or CNT bundles, and that the influence of crystallinity is only visible when near-perfect alignment and purity are achieved. The low-temperature resistivity decreases with increasing temperature (Figure 3), indicating that all films behave primarily as semiconductors [57] [23] [58] and only show a metallic upturn above the cross-over temperature $T^{*} \sim 220-250 \mathrm{~K}$.

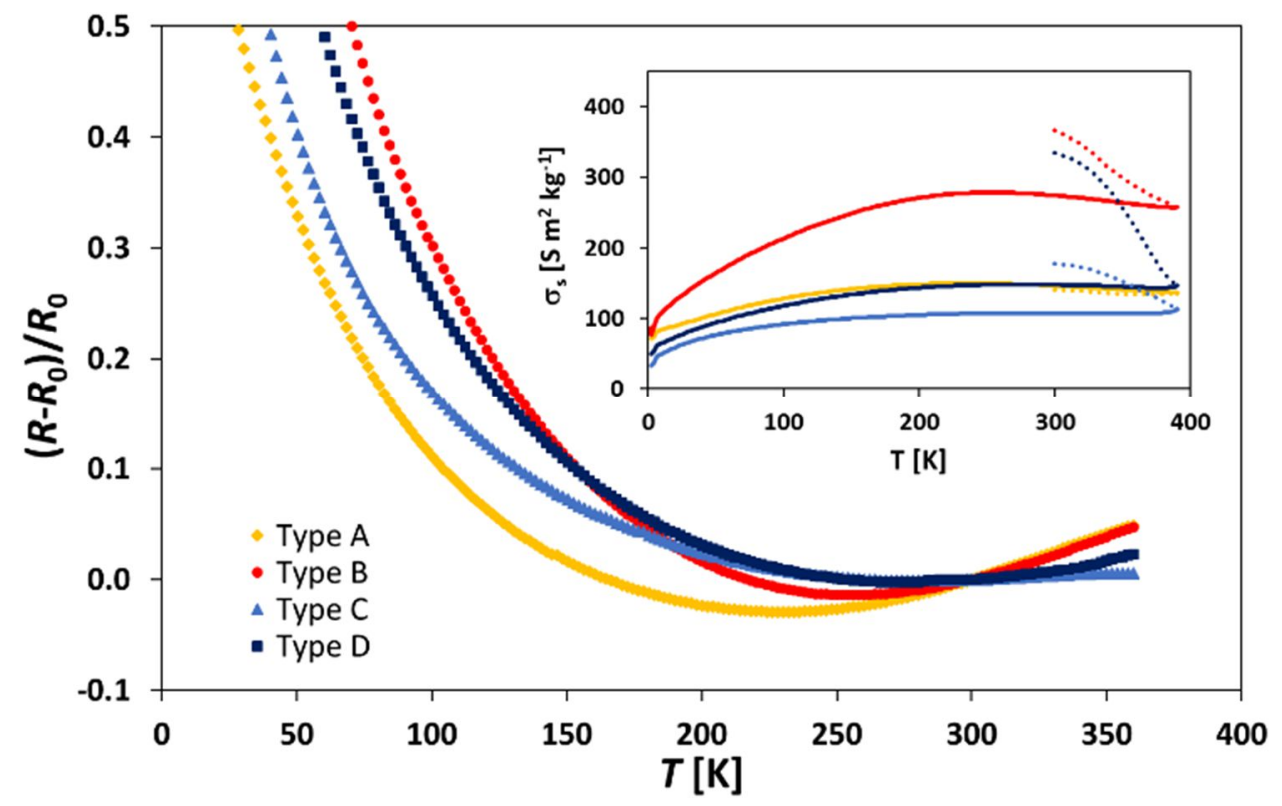

Figure 3: Reduced electrical resistivity normalised to room temperature resistivity from each of the three specimens per sample type. The low-temperature resistivity of all samples decreases with increasing temperature, which is typical for mostly semiconducting materials, with only a low positive slope above the cross-over temperature at $\sim 250 \mathrm{~K}$. The inset shows the specific electrical conductivity data with the typical outgassing hysteresis during annealing. (Absolute electrical conductivity data in Supp. Inf. S4)

Electrical conduction strongly depends on whether the CNT materials are measured 'as-is', or whether there was an outgassing step prior to the actual measurement as in the $\sigma(\mathrm{T})$ measurements presented herein. During this initial heating phase, evacuation and annealing to $390 \mathrm{~K}$, water and physisorbed oxygen which are responsible for the p-type conduction of CNTs are removed from the surface and the electrical conductivity is decreased significantly (dotted lines in Figure 3 inset). Similar temperature-dependent electrical conductivity hysteresis was reported previously [59].

Although there appears to be a large difference between the different types of samples in absolute conductivity (Supp. Inf. S4), these differences are primarily caused by the different thicknesses of the samples and different linear density, and disappear if the data are presented in specific units per unit density.

Only Type B samples with specific electrical conductivities of approximately $400 \mathrm{~S} \mathrm{~m}^{2} \mathrm{~kg}^{-1}$ at ambient conditions, and $280 \mathrm{~S} \mathrm{~m}^{2} \mathrm{~kg}^{-1}$ after outgassing, show a significantly higher conductivity 
than the other samples, both specific and in absolute terms (SI). We attribute this to the overall better contact between CNTs due to their alignment, as well as to the CNT bundle coating, which concurs with observations made with CNT fibres infiltrated with pyrocarbons [56]. Given the precursor, we postulate that since the coating contains mainly aromatic groups, which are conformationally restricted on surface of nanotubes by pi-stacking interactions, it would not impede electron conduction between nanotubes as much as an amorphous carbon coating. Other explanations could include a difference in CNT length between the samples, however, there is no method for mean length measurements in bulk CNT material, and we exclude this possibility for now.

\subsection{Thermal Conductivity of CNT Films}

The film samples spun from different carbon precursors exhibit very different apparent thermal conductivities in terms of their magnitude (Supp. Inf. S5). The high-purity SWCNT sample spun from n-butanol (Type D) is in absolute terms the poorest thermal conductor, followed by the SWCNT sample containing cluster impurities (Type C), while Type A and B are an order of magnitude better. Again, this effect originates from the low film thickness and weight in both cases, and after accounting for the different densities (Figure 4), all CNT films show more comparable specific conductivities, which implies that the absolute conductivity was mainly influenced by sample mass.

If reported in specific terms, the thermal conductivity of Type A is below all the other samples, which means that its high absolute thermal conductivity was due to more material being present. As the sample is mainly comprised by multi-wall tubes, one would expect a thermal conductivity lower than for single-walled or mixed samples. Type C's thermal conductivity is virtually equivalent to Type A, although the composition of the samples is very different. We conclude that the bundlebundle contact of Type $\mathrm{C}$ is deteriorated by the presence of cluster impurities of deformed MWCNTs which increases the thermal junction resistance.

Qualitatively, the shape of the temperature dependence of the thermal conductivity for Types A, C and D is distinctively convex compared to Type B sample's rather linear behaviour. A log-log plot of the absolute thermal conductivities (Figure 4 inset) reveals a power law behaviour as previously described [21], with the three samples Type A, C and D following a comparable behaviour $\sim T^{\alpha}$, with $\alpha=1.8,1.7$ and 1.9, respectively, while Type B shows a significantly lower coefficient 
$\alpha=1.0$. A smaller exponent $\alpha$ corresponds to lower dimensionality in phonon heat conduction and can be attributed to higher bundle alignment or better inter-bundle contact ( [23], [20], [38], [36], [53]), which is in agreement with the observations by SEM or SAXS. For $50 \mathrm{~K}$, we note that the measurement of Type B diverts from this power law. According to [20] and [21], the Debye temperature at $\sim 50 \mathrm{~K}$ marks a transition at which the low-dimensional character due to fully occupied interlayer phonons changes to a high-dimensional character at lower temperatures.

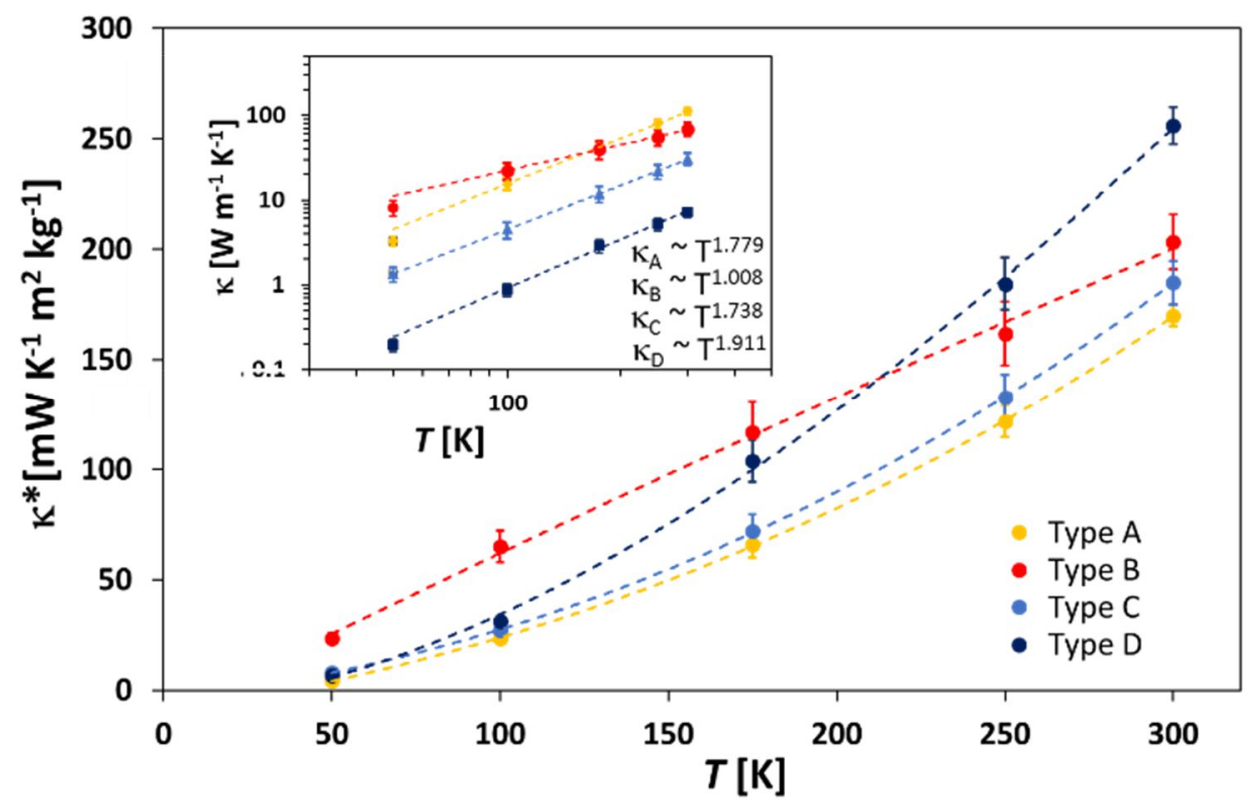

Figure 4: Specific thermal conductivity data averaged from each three measurements per sample type. Inset: Thermal conductivity plotted on log-log scale indicates that the samples follow a power law down above $100 \mathrm{~K}$, with Type B showing a significantly low-dimensional transport character.

Although Type B thermal conductivity observations concur with Type B also being superior in electrical conductivity, the other samples' thermal conductivity does not follow the same trend as electrical conductivity. We estimated the electrical contribution to the thermal conductivity from the effective Lorentz number defined by the Wiedemann-Franz as $L_{\text {eff }}=\kappa / \sigma T$, with the electrical conductivity $\sigma$, and temperature $T$ [23] [60] [51] [15]. $L_{\text {eff }}$ is for all samples more than two orders of magnitude larger than the Lorentz number for metals $L_{\mathrm{m}}=2.45 \cdot 10^{-8}(\mathrm{~V} / \mathrm{K})^{2}$, indicating that the heat conduction is indeed dominated by phonons rather than electrons. Interestingly, the electrical contribution of Type B is almost double of that of the other samples.

Initially counterintuitive, Type D shows the highest thermal conductivity close to room temperature with the steepest thermal gradient (Figure 4) despite the poorest alignment which would be expected to correspond to a lowered mechanical stiffness. A tentative explanation for this 
phenomenon could be that the specific thermal conductivity falls with increasing density as shown in Figure 5. Apart from Type C, whose dependence on density is deteriorated by the cluster impurities pushing the aligned CNT bundles apart, samples A, B and D follow a linear trend. This supports the suggestion by [30] that contact points between CNT bundles occurring at intervals with a certain distance are preferable to continuous contact to minimise the dampening effect by intertube scattering. The low density of sample D counteracts the effect of the poor alignment. In turn this also underlines why the conductivity increase in Type B is lower than for the other samples, as here the carbonaceous bundle coating leads to more coupling between the CNT bundles and a higher dampening effect with increasing temperature. Further studies regarding the effect of bundle coupling by carbonaceous coating or number of contact points need to follow.

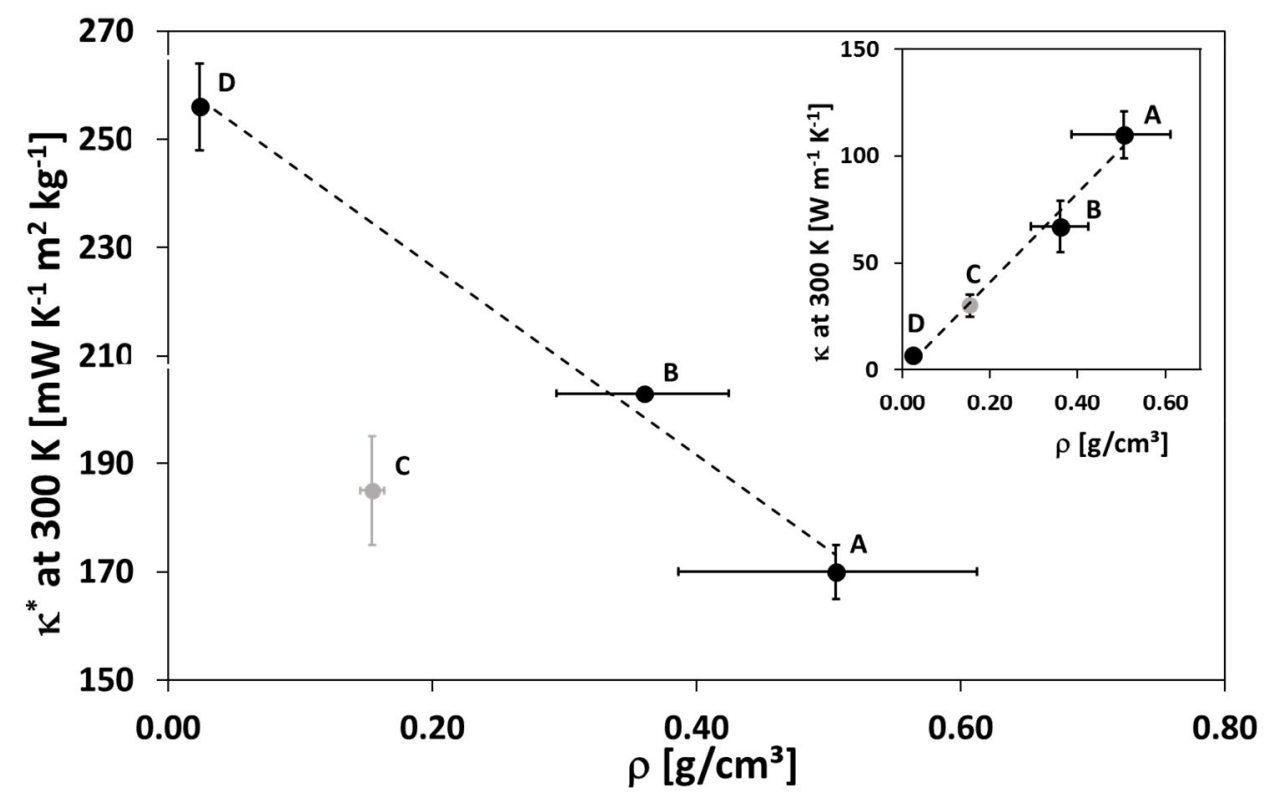

Figure 5: The specific conductivity at $300 \mathrm{~K}$ decreases for samples Type A, B and D with film density, Type C deviates from this dependence due to the cluster impurities. The inset shows the absolute values, which, as expected, increase with the amount of material present. Again, the importance of using specific data is emphasised, as the absolute values of conductivity simply increase with weight of the films, while the specific data show the quality of the material.

\subsection{Thermal Conductivity of Micro-Fibres}

Thermal conductivity measurements were also conducted on micro-fibre samples spun from methane (Type A) and toluene (Type B). The synthesised material was required to be mechanically strong enough during spinning to withstand both the online condensation and stretching between two winders, and subsequently the testing procedure. Compared to the film fabrication, where 
condensation mainly effects the thickness and hence out-of-plane bundle contact and alignment (one-dimensional condensation), micro-fibres are condensed radially (two-dimensional condensation) which, together with the stretching, leads to a much improved alignment of the bundles. The linear densities and cross sectional areas of the measured micro-fibres are summarised Supp. Inf., Table S9.

The specific thermal conductivities of the Type $A$ and $B$ fibres at $300 \mathrm{~K}$ were $970 \pm 50 \mathrm{~mW} \mathrm{~K}^{-1} \mathrm{~m}^{2} \mathrm{~kg}^{-1}$, and $1220 \pm 40 \quad \mathrm{~mW} \mathrm{~K}^{-1} \mathrm{~m}^{2} \mathrm{~kg}^{-1}$, respectively. These thermal conductivities, both specific and absolute terms, are the highest reported to date for as-spun pristine CNT fibres [39] [59], even higher than for acid-treated or iodine-doped fibres [24].

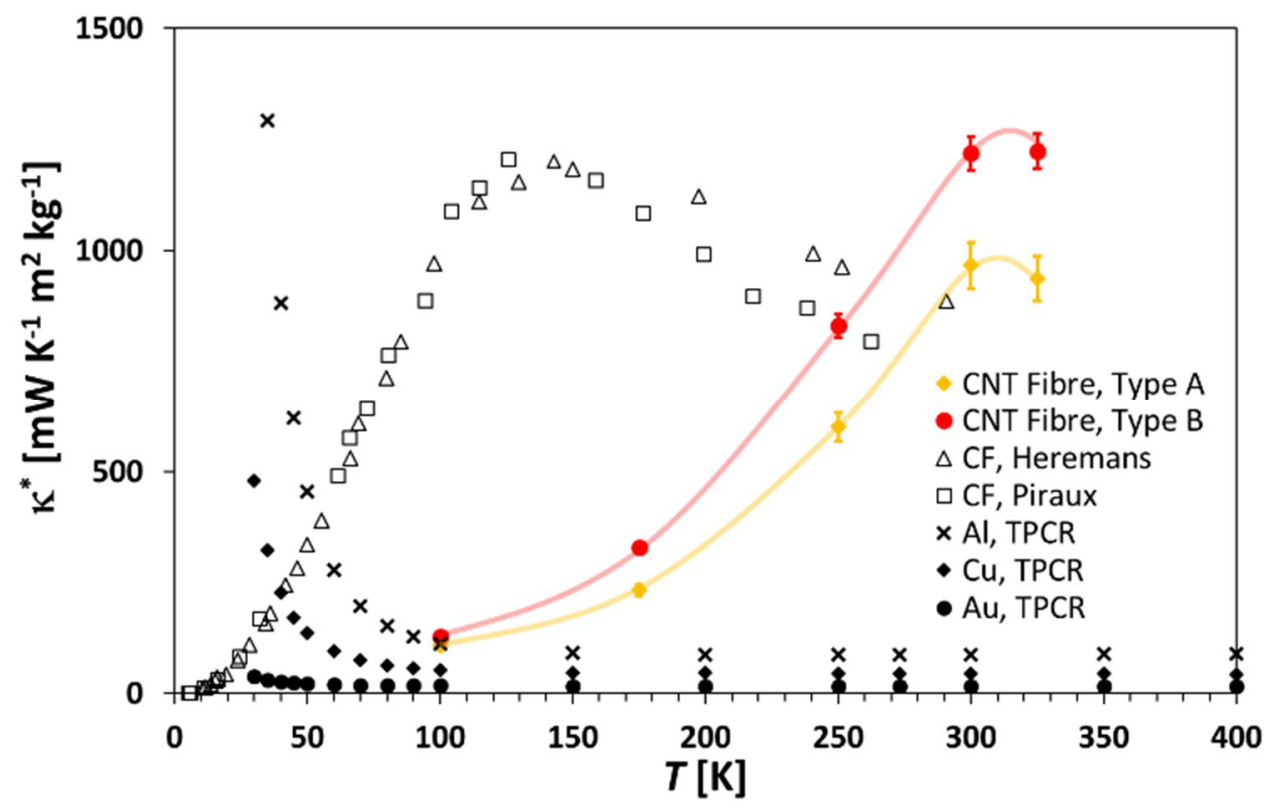

Figure 6: The specific thermal conductivities of Type A (yellow circles) and Type B (red circles) micro-fibres at $300 \mathrm{~K}$ are with $970 \pm 50$ and $1220 \pm 40 \mathrm{~mW} \mathrm{~K}^{-1} \mathrm{~m}^{2} \mathrm{~kg}^{-1}$, respectively, the highest values reported for pristine dry-spun CNT fibres to date. (Lines shown are guides to the eye.) The maximum thermal conductivities of the fibres are on a par with those of heat-treated carbon fibres (open triangles and squares) [11] [13], and 50\% higher at room temperature. The values for thermal conductivities of metals (crosses, black lozenges, circles) from TPCR [18] depict results for bulk materials rather than fibres so serve only for qualitative comparison.

Both fibres show evidence of a broad peak in $\kappa(T)$ above $300 \mathrm{~K}$ (Figure 6), as has been observed previously [23] [14] [20] [24]. According to the intrinsic thermal conductivity behaviour described in [16] [17], this peak marks the onset of Umklapp mechanisms, a result of thermally activated phonon activity. 
It is known for carbon fibre [61] that a higher thermal conductivity goes together with a higher elastic modulus. The elastic moduli of Type $\mathrm{A}$ and $\mathrm{B}$ is 41 and $78 \mathrm{~N} \mathrm{tex}^{-1}$, respectively, which indicates that these fibres, despite being a yarn-like material, follow a similar trend.

The thermal conductances of these small-diameter CNT fibres were only $10-15 \%$ of the background conductance, which rendered accurate measurements of $\kappa$ below $100 \mathrm{~K}$ impossible [51] [54]. Therefore, it was also not possible to estimate the mean free paths at very low temperatures when the phonon states are frozen out [15].

The absolute thermal conductivities of Type $\mathrm{A}$ and $\mathrm{B}$ fibres, $630 \pm 30 \mathrm{~W} \mathrm{~m}^{-1} \mathrm{~K}^{-1}$, and $770 \pm 10 \mathrm{~W} \mathrm{~m}^{-1} \mathrm{~K}^{-1}$, respectively at room temperature, were determined using several focused ion beam cut cross-sectional areas of these yarns (Supp. Inf. S5). Being condensed by acetone spray without any twisting, the micro-fibres are not of a circular cross section. Often, the condensation leads to an involuted ribbon-like morphology that leads to significant overestimation (up to a factor of five) of circular cross section estimated from measurement of the outer diameter. A similar matter has been reported by other authors for related CNT materials [62]. For micro-fibres, even more so than for thin films, specific thermal conductivity using the linear density gives a far more reliable value for the overall performance of the fibre given that the linear density is measured from $90 \mathrm{~mm}$ strands and averaged from three strands. Even if the cross sections are diligently measured and averaged from several cut sections, which is highly time consuming, it remains an average of discrete positions along the fibre. Additionally, a reduction in cross-sectional area, such as by twisting, is meaningless if it does not lead in fact to a higher thermal conductivity in specific terms, showing that the same amount of material indeed gives improved values, e.g. by better bundlebundle contact.

The thermal conductivity of fibres is, in both absolute and specific terms, far superior to that of films, due to (1) the smaller amount of CNTs and bundles and (2) the improved bundle alignment. As there is a trade-off between increasing the number of nanotubes and the resultant increase of junctions [30], a larger number of CNTs will increase the overall thermal conductivity, but the dominance of junctions will cause a decrease in conductivity of the bulk material. Improved bundle alignment would cause better contact and higher overlap area between the CNTs, increasing the thermal conductivity [53] [23] [36] [62].

Figure 7 shows the specific thermal conductivities of the presented films and fibres as a function of the cross sectional area, in comparison to previous reports. Although the films showed only $\sim 30 \%$ of the thermal conductivity of the online condensed fibres, all present values are far above 
values reported for other similar materials of similar cross sections, independent of whether they contain predominantly single- or multi-walled CNTs, or their state of alignment.

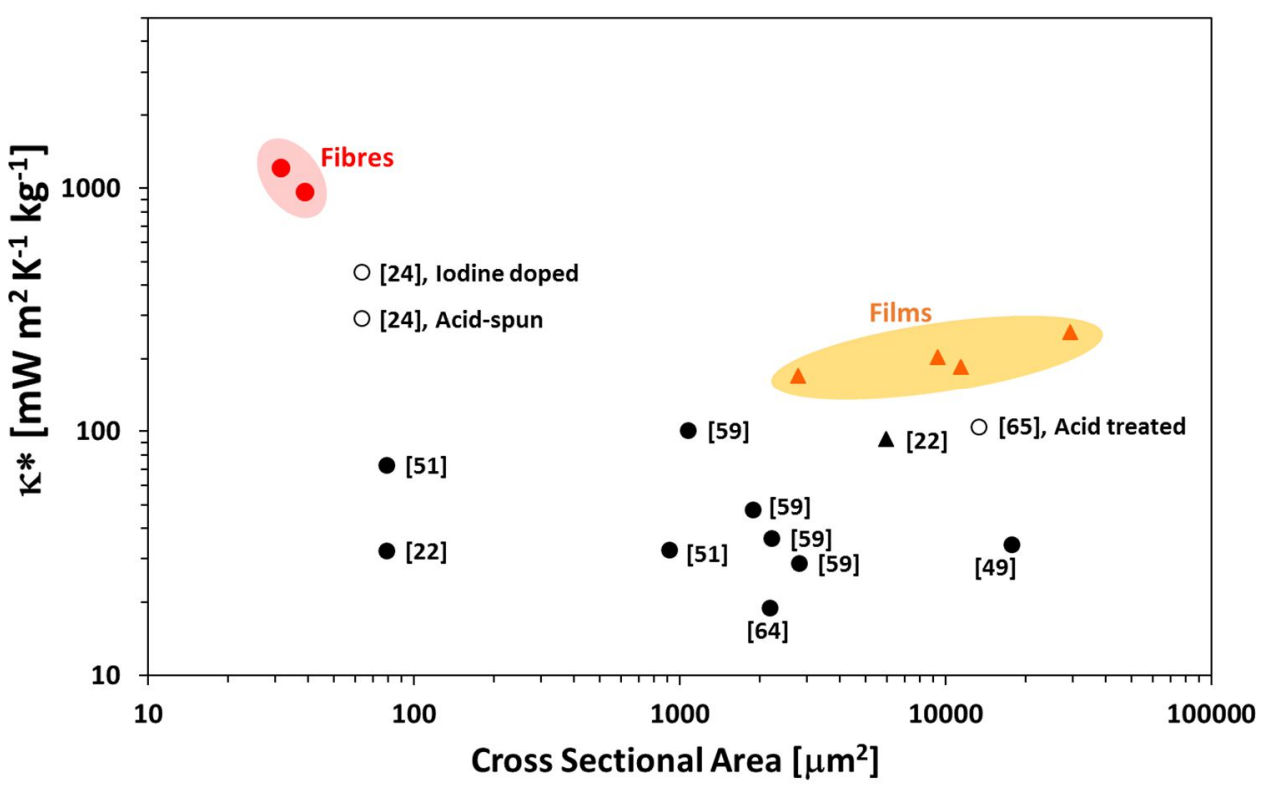

Figure 7: Specific thermal conductivity as a function of cross sectional area at room temperature. Black circles and triangles refer to pristine CNT fibres or films, respectively, while empty circles refer to post-processed CNT fibres, such as acid-treated or iodine-doped. Red circles and triangles refer to the fibres and films presented in this work. (The density of fibres used in [59] was measured here as $0.52 \mathrm{~g} \mathrm{~cm}^{-3}$.)

According to [22] [63] [14] [32], the longer the nanotubes, the steeper the temperature gradient of the thermal conductivity, and nanotubes that are sufficiently longer than the phonon mean free path are less sensitive to bending [25], as low-frequency phonons do not appear to be scattered by kinks. The effects of alignment for the different samples discussed here relate to better contact between the CNT bundles in Type B or in fibres compared to films, but all of the samples have - as typical for floating catalyst CVD spun CNTs - lengths of the order of several hundred microns [41], which would explain the overall higher thermal conductivity.

\section{Conclusion}

We presented thermal conductivity of CNT fibres and films, directly spun from the gas phase, showing the highest thermal conductivities for CNT bulk materials reported to date. Four sample types were compared, ranging from predominantly single- to multi-walled, well-aligned or virtually unaligned, pure to containing impurities. For both electrical and thermal conductivity, we show that at least for the as-spun material, density and alignment are more essential than whether the CNTs 
are single-walled or multi-walled. In terms of purity, larger impurities such as cluster impurities disrupt bundle contact and decrease conductivity. The classification of carbonaceous bundle coating depends on the carbon precursor used and can range from being simply an impurity, to acting as fortifying agent - similar to a matrix in a composite - helping both bundle contact, and also increasing the electrical conductivity and leading to a lower temperature coefficient of thermal conductivity. Due to the two-dimensional condensation of fibres, the thermal conductivity was increased by as much as a factor of 6 compared to the same material in film morphology. For effective heat dissipation applications, extrinsic effects, such as alignment and morphological purity, have to be controlled before effects of CNT character and crystallinity will be visible.

\section{Acknowledgements}

The authors thank W. Skelton-Hough, and Dr A. Mikhalchan for assisting with the fibre spinning which is still a two-person process, and Dr J. Bulmer for laser-cutting of the CNT films and discussions regarding electrical conduction mechanisms. We also acknowledge the US Office of Naval Research (W911NF-11-1-0250 and ONR Global (N62909-15-1-2034) for funding and support. The work at Dalhousie University was supported by NSERC, as well as the Canada Foundation for Innovation, Atlantic Innovation Fund, Dalhousie University and other partners that fund the Facilities for Materials Characterization managed by the Institute for Research in Materials.

\section{References}

[1] Q. Zhang, J.-Q. Huang, M.-Q. Zhao, W.-Z. Qian and F. Wei, "Carbon nanotube mass production: Principles and processes," ChemSusChem, vol. 4, p. 864-889, 2011.

[2] J.-B. Donnet, R. C. Bansal and M.-J. Wang, Carbon Black: Science and Technology, New York: Marcel Dekker, 1993.

[3] V. B. Gupta and V. K. Kothari, Manufactured Fibre Technology, London: Chapman \& Hall, 1997.

[4] D. D. L. Chung, Carbon Fiber Composites, Newton, MA: Butterworth-Heinemann, 1994.

[5] S.-J. Park, Carbon Fibers, Dordrecht: Springer, 2015. 
[6] R. Saito, G. Dresselhaus and M. S. Dresselhaus, Physical Properties of carbon nanotubes, London: Imperial college press, 1998.

[7] J.-C. Charlier, X. Blase and S. Roche, "Electronic and transport properties of nanotubes," Reviews of Modern Physics, vol. 79, pp. 677-732, 2007.

[8] P. J. Burke, C. Rutherglen and Z. Yu, Nanotubes and Nanowires, P. J. Burke, Ed., Singapore: World Scientific Publishing, 2007.

[9] A. Lekawa-Raus, J. Patmore, L. Kurzepa, J. Bulmer and K. Koziol, "Electrical properties of carbon nanotube based fibers and their future use in electrical wiring," Advanced Functional Materials, vol. 24, p. 3661-3682, 2014.

[10] T. R. Anthony, W. F. Banholzer, J. F. Fleischer, LanhuaWei, P. K. Kuo et al., "Thermal diffusivity of isotopically enriched C12 diamond," Physical Reviews B 42, p. 1104-1111, 1990.

[11] L. Piraux, B. Nysten, A. Haquenne, J.-P. Issi, M. Dresselhaus and M. Endo, "The temperature variation of the thermal conductivity of benzene-derived carbon fibers," Solid State Communications, vol. 50, no. 8, p. 697-700, 1984.

[12] P. M. Adams, H. A. Katzman, G. S. Rellick and G. W. Stupian, "Characterization of high thermal conductivity carbon fibers and a self-reinforced graphite panel," Carbon, vol. 36, no. 3, 233-245, 1998.

[13] J. Heremans and J. C. P. Beetz, "Thermal conductivity and thermopower of vapor-grown graphite fibers," Physical Review B, vol. 32, p. 1981-1986, 1985.

[14] E. Pop, D. Mann, Q. Wang, K. Goodson and H. Dai., "Thermal Conductance of an Individual Single-Wall Carbon Nanotube above Room Temperature," Nano Letters, vol. 6, no. 1, p. 96$100,2006$.

[15] J. Hone, M. Whitney, C. Piskoti and A. Zettl., "Thermal conductivity of singlewalled carbon nanotubes," Physical Review B, vol. 59, no. 4, p. 2514-2516, 1999.

[16] S. Berber, Y.-K. Kwon and D. Tomanek., "Unusually high thermal conductivity of carbon nanotubes," Physical Review Letters, vol. 84, no. 20, p. 4613-4616, 2000.

[17] N. Mingo and D. A. Broido, "Carbon Nanotube Ballistic Thermal Conductance and Its Limits," Physical Review Letters, vol. 95, p. 096105, 2005.

[18] Y. Touloukian, R. Powell, C. Ho and P. Klemens, Thermophysical Properties of Matter - The TPRC Data Series, Vols. 1. Thermal Conductivity-Metallic Elments and Alloys, New York: 
Plenum Publishing Corp, 1970.

[19] A. M. Marconnet, M. A. Panzer and K. E. Goodson., "Thermal conduction phenomena in carbon nanotubes and related nanostructured materials," Reviews of Modern Physics, vol. 85, p. 1295-1326, 2012.

[20] P. Kim, L. Shi, A. Majumdar and P. L. McEuen, "Thermal Transport Measurements of Individual Multiwalled Nanotubes," Physical Review Letters, vol. 87, no. 21, p. 215502, 2001.

[21] R. Jin, Z. Zhou, D. Mandrus, I. Ivanov, G. Eres, J. Howe et al., "The effect of annealing on the electrical and thermal transport properties of macroscopic bundles of long multi-wall carbon nanotubes," Physica B, vol. 388 , p. 326-330, 2007.

[22] A. E. Aliev, C. Guthy, M. Zhang, S. Fang and A. A. Zakhidov, "Thermal transport in MWCNT sheets and yarns," Carbon, vol. 45, p. 2880-2888, 2007.

[23] J. Hone, M. C. Llaguno, N. M. Nemes, A. T. Johnson, J. E. Fischer, D. A. Walters et al., "Electrical and thermal transport properties of magnetically aligned single wall carbon nanotube films," Applied Physics Letters, vol. 77, p. 666-668, 2000.

[24] N. Behabtu, C. C. Young, D. E. Tsentalovich, O. Kleinerman, X. Wang, A. W. K. Ma et al., "Strong, Light, Multifunctional Fibers of Carbon Nanotubes with Ultrahigh Conductivity," Science, vol. 339, no. 6116, p. 182-186, 2013.

[25] J. Ma, Y. Ni and T. Dumitrică, "Thermal conductivity and phonon scattering in severely bent carbon nanotubes and bi-layer graphene," Materials Today: Proceedings, vol. 2, p. 3819-3823, 2015.

[26] J. Che, T. Cagin and W. A. Goddard, "Thermal conductivity of carbon nanotubes," Nanotechnology, vol. 11 , p. 65-69, 2000.

[27] C. Chang, D. Okawa, H. Garcia, A. Majumdar and A. Zettl, "Breakdown of Fourier's Law in Nanotube Thermal Conductors," Physical Review Letters, vol. 101, p. 075903, 2008.

[28] M. Mir, E. Ebrahimnia-Bajestan, H. Niazmand and M. Mir, "A novel approach for determining thermal properties of single-walled carbon nanotubes," Computational Materials Science, vol. 63, p. 52-57, 2012.

[29] X. Zhang, W.-X. Zhou, X.-K. Chen, Y.-Y. Liu and K.-Q. Chen, "Significant decrease in thermal conductivity of multi-walled carbon nanotube induced by inter-wall van der Waals interactions," Physics Letters A, vol. 380, p. 1861-1864, 2016. 
[30] A. E. Aliev, M. H. Lima, E. M. Silverman and R. H. Baughman, "Thermal conductivity of multi-walled carbon nanotube sheets: radiation losses and quenching of phonon modes," Nanotechnology, vol. 21 , p. 035709, 2010.

[31] B. Yakobson and L. Couchman, "Persistence length and nanomechanics of random bundles of nanotubes," Journal of Nanoparticle Research, vol. 8, p. 105-110, 2006.

[32] H. Zhong and J. R. Lukes, "Interfacial thermal resistance between carbon nanotubes: Molecular dynamics simulations and analytical thermal modeling," Physical Review B, vol. 74, p. $125403,2006$.

[33] F. Xu, A. Sadrzadeh, Z. Xu and B. I. Yakobson, "Can carbon nanotube fibers achieve the ultimate conductivity? - Coupled-mode analysis for electron transport through the carbon nanotube contact," Journal of Applied Physics, vol. 114, p. 063714 , 2013.

[34] T. S. Gspann, N. Montinaro, A. Pantano, J. A. Elliott and A. H. Windle, "Mechanical properties of carbon nanotube fibres: St Venant's principle at the limit and the role of imperfections," Carbon, vol. 93, p. 1021-1033, 2015.

[35] J. J. Vilatela, J. A. Elliott and A. H. Windle, "A model for the strength of yarn-like carbon nanotube fibers," ACS Nano, vol. 5, no. 3, p. 1921-1927, 2011.

[36] P. Gonnet, Z. Liang, E. S. Choi, R. S. Kadambala, C. Zhang, J. S. Brooks et al., "Thermal conductivity of magnetically aligned carbon nanotube buckypapers and nanocomposites," Current Applied Physics, vol. 6, p. 119-122, 2006.

[37] D. Wang, P. Song, C. Liu, W. Wu and S. Fan, "Highly oriented carbon nanotube papers made of aligned carbon nanotubes," Nanotechnology, vol. 19 , p. 075609, 2008.

[38] J. E. Fischer, W. Zhou, J. Vavro, M. C. Llaguno, C. Guthy, R. Haggenmueller et al., "Magnetically aligned single wall carbon nanotube films: Preferred orientation and anisotropic transport properties," Journal of Applied Physics, vol. 93, p. 2157-2163, 2003.

[39] E. Mayhew and V. Prakash, "Thermal conductivity of high performance carbon nanotube yarnlike fibers," Journal of Applied Physics, vol. 115, p. 174306, 2014.

[40] H. Misak and S. Mall, "Investigation into microstructure of carbon nanotube multi-yarn," Carbon, vol. 72 , p. 321-327, 2014.

[41] K. Koziol, J. Vilatela, A. Moisala, M. Motta, P. Cunniff, M. Sennett et al., "High-Performance Carbon Nanotube Fiber,” Science, vol. 318, no. 5858, p. 1892-1895, 2007. 
[42] T. Gspann, F. Smail and A. Windle, "Spinning of carbon nanotube fibres using the floating catalyst high temperature route: purity issues and the critical role of sulphur.," Faraday Discussions, vol. 173, p. 47-65, 2014.

[43] Y.-L. Li, I. A. Kinloch and A. H. Windle, "Direct Spinning of Carbon nanotube fibres from chemical vapour deposition synthesis," Science, vol. 304, no. 5668, p. 276-278, 2004.

[44] M. Motta, I. Kinloch, A. Moisala, V. Premnath, M. Pick and A. Windle, "The parameter space for the direct spinning of fibres and films carbon nanotubes," Physica E, vol. 37 , p. $40-43$, 2007.

[45] Y. Jung, J. Song, W. Huh, D. Cho and Y. Jeong, "Controlling the crystalline quality of carbon nanotubes with processing parameters from chemical vapor deposition synthesis," Chemical Engineering Journal, vol. 228, p. 1050-1056, 2013.

[46] V. Reguero, B. Alemán, B. Mas and J. J. Vilatela, "Controlling carbon nanotube type in macroscopic fibers synthesized by the direct spinning process," Chemistry of Materials, vol. 26, p. 3550-3557, 2014.

[47] D. Conroy, A. Moisala, S. Cardoso, A. Windle and J. Davidson, "Carbon nanotube reactor: Ferrocene decomposition, iron particle growth, nanotube aggregation and scale-up," Chemical Engineering Science, vol. 65, p. 2965-2977, 2010.

[48] S. Li, X. Zhang, J. Zhao, F. Meng, G. Xu, Z. Yong et al., "Enhancement of carbon nanotube fibres using different solvents and polymers," Composites Science and Technology, vol. 72 , p. 1402-1407, 2012.

[49] J. Qiu, J. Terrones, J. J. Vilatela, M. E. Vickers, J. A. Elliott and A. H. Windle, "Liquid Infiltration into Carbon Nanotube Fibers: Effect on Structure and Electrical Properties," ACS Nano, vol. 7 , no. 10, p. 8412-8422, 2013.

[50] J.-H. Pohls, M. B. Johnson, M. A. White, R. Malik, C. J. B. Ruff, M. J. Schulz et al., "Physical properties of carbon nanotube sheets drawn from nanotube arrays.," Carbon, vol. 50, no. 11, p. 4175-4183, 2012.

[51] M. B. Jakubinek, M. B. Johnson, M. A. White, C. Jayasinghe, W. C. G. Li, M. J. Schulz et al., "Thermal and electrical conductivity of array-spun multi-walled carbon nanotube yarns," Carbon, vol. 50, p. 244-248, 2012.

[52] M. Jakubinek, J. F. Niven, M. B. Johnson, B. Ashrafi, K. S. Kim, B. Simard et al., "Thermal 
conductivity of bulk boron nitride nanotube sheets and their epoxy-impregnated composites," Physica Status Solidi A, no. 213, p. 2237-2242, 2016.

[53] S. Badaire, V. Pichot, C. Zakri, P. Poulin, P. Launois, J. Vavro et al., "Correlation of properties with preferred orientation in coagulated and stretch-aligned single-wall carbon nanotubes," Journal of Applied Physics, vol. 96, no. 12, p. 7509-7513, 2004.

[54] B. M. Zawilski, R. T. Littleton and T. M. Tritt, "Description of the parallel thermal conductance technique for the measurement of the thermal conductivity of small diameter samples," Review of Scientific Instruments, vol. 72, no. 3, p. 1770-1774, 2001.

[55] S. Boncel, R. M. Sundaram, A. H. Windle and K. K. Koziol, "Enhancement of the Mechanical Properties of Directly Spun CNT Fibers by Chemical Treatment," ACS Nano, vol. 5, no. 12, p. 9339-9344, 2011.

[56] V. Thiagarajan, X. Wang, P. Bradford, Y. Zhu and F. Yuan, "Stabilizing carbon nanotube yarns using chemical vapor infiltration," Composites Science and Technology, vol. 90 , p. 82 87, 2014.

[57] A. B. Kaiser, G. Düsberg and S. Roth, "Heterogeneous model for conduction in carbon nanotubes," Physical Review B, vol. 57, no. 3, p. 1418-1421, 1998.

[58] Y. Zhao, J. Wei, R. Vajtai, P. Ajayan and E. Barrera, "Iodine doped carbon nanotube cables exceeding specific electrical conductivity of metals," Scientific Reports, vol. 1, p. 1-5, 2011.

[59] J. F. Niven, M. B. Johnson, S. M. Juckes, M. White, N. Alvarez and V. Shanov, "Influence of annealing on thermal and electrical properties of carbon nanotube yarns.," Carbon, vol. 99, p. 485-490, 2016.

[60] D. Yang, S. Wang, Q. Zhang, P. Sellin and G. Chen, "Thermal and electrical transport in multiwalled carbon nanotubes," Physics Letters A, vol. 329 , p. 207-213, 2004.

[61] F. Emmerich, "Young's modulus, thermal conductivity, electrical resistivity and coefficient of thermal expansion of mesophase pitch-based carbon fibers," Carbon, p. 274-293, 2014.

[62] W. Zhou, J. Vavro, C. Guthy, K. I. Winey, J. E. Fischer, L. M. Ericson et al., "Single wall carbon nanotube fibers extruded from super-acid suspensions: Preferred orientation, electrical, and thermal transport," Journal of Applied Physics, vol. 95, p. 649-655, 2004.

[63] M. Wang and Z.-Y. Guo, "Understanding of temperature and size dependences of effective thermal Understanding of temperature and size dependences of effective thermal," Physics 
Letters A, vol. 374 , p. 4312-4315, 2010.

[64] L. M. Ericson, H. Fan, H. Peng, V. A. Davis, W. Zhou, J. Sulpizio et al., "Macroscopic, neat, single-walled carbon nanotube fibers," Science, vol. 305, p. 1447-1450, 2004.

[65] P. Liu, Z. Fan, A. Mikhalchan, T. Q. Tran, D. Jewell, H. M. Duong and A. M. Marconnet, "Continuous carbon nanotube-based fibers and films for applications requiring enhanced heat dissipation,” ACS Applied Materials \& Interfaces, vol. 8, p. 17461-17471, 2016. 